\title{
Iatrogenic superior vena cava syndrome from percutaneous right ventricular assist device
}

\author{
Bernice Badu, BA, Lucian Durham III, MD, PhD, Lyle D. Joyce, MD, PhD, and David L. Joyce, MD, \\ Milwaukee, Wis
}

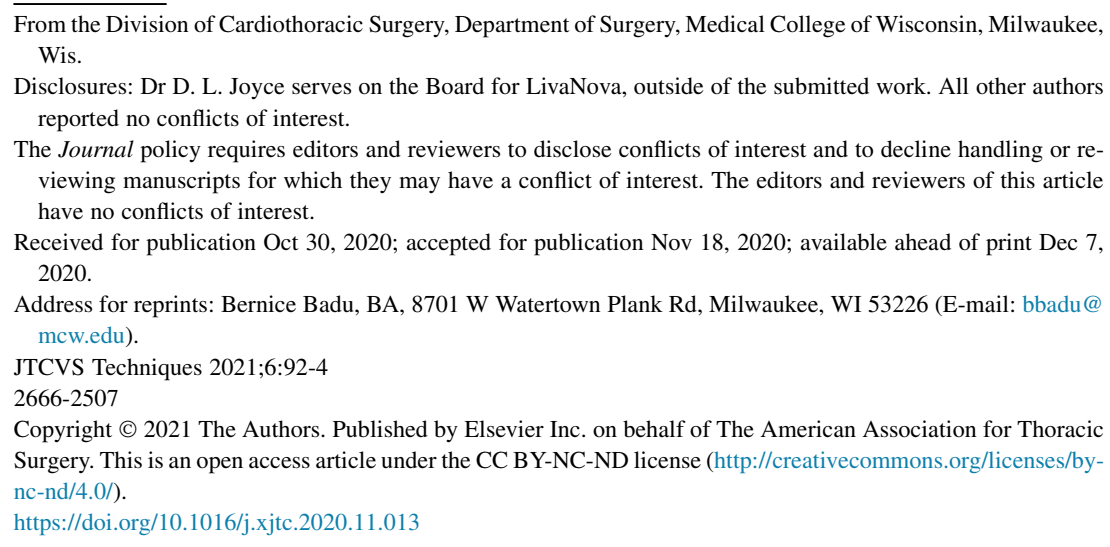

Video clip is available online.

Percutaneous right ventricular assist device (RVAD) support via right internal jugular (RIJ) vein access with a dual lumen cannula (DLC) has emerged as a viable solution for treating refractory right ventricular (RV) failure..$^{1-4}$ Despite these benefits, the safety profile of such devices has not been fully characterized. We present 2 of 3 patients who developed superior vena cava (SVC) syndrome following percutaneous RVAD support and discuss the postoperative management of this complication.

\section{CLINICAL SUMMARY \\ Patient 1}

A 54-year-old female patient with a history of ischemic cardiomyopathy and acute coronary syndrome, status-post coronary bypass grafting, presented for implantation of a durable left ventricular assist device (LVAD; HeartWare HVAD, Medtronic, Inc, Minneapolis, Minn). Following LVAD implant, she required a 31-Fr cannula RVAD (TandemLife ProtekDuo; LivaNova, Pittsburgh, Pa) for RV dysfunction and respiratory failure from pulmonary hypertension. The cannula was placed in this patient whose SVC length-from the brachiocephalic confluence to the atriocaval junction-measured $52 \mathrm{~mm}$ compared with a mean (standard deviation) of 63.9 (13.5) $\mathrm{mm}$ of the entire cohort of patients supported with this size cannula (Table 1).

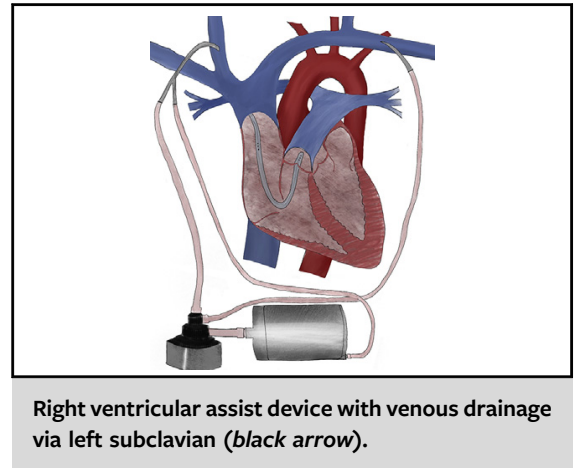

\begin{abstract}
CENTRAL MESSAGE
Although SVC syndrome is a lifethreatening complication from RVADs, we developed a technique that rapidly resolves symptoms and permits patients to remain on support long enough for RV recovery.
\end{abstract}

See Commentaries on pages 95 and 97.
Overnight, the patient was noted to have developed SVC syndrome secondary to functional obstruction from the RVAD cannula in her relatively shorter SVC. There was no evidence of thrombosis of the SVC or central veins, and she did not have a history of malignancy or venothromboembolism. To manage this complication, a 9.5 sheath was advanced through the side port of the pulmonary arterial catheter and into the patient's left subclavian vein (Figure 1). The other end of the sterile tubing was attached to the DLC circuit, which acted as a negative pressure to drain venous blood. This served as a "blow off" safety valve for venous congestion, leading to reduction of upper extremity and face edema.

Following intervention, there was rapid resolution of cyanosis and edema of her face and neck, and she tolerated $\mathrm{RV}$ support well. The remainder of her hospital course was complicated by multisystem organ failure and respiratory failure requiring tracheostomy. There was no improvement in her clinical status after 43 days, and the patient's family 
TABLE 1. Outcomes and characteristic of patients with SVC syndrome following right ventricular support with percutaneous dual-lumen RVAD, compared with entire cohort

\begin{tabular}{lcccc}
\hline & Patient 1 & Patient 2 & Patient3 & All patients, N $=\mathbf{4 0}$ \\
\hline SVC syndrome & Yes & Yes & Yes & $3(7.5 \%)$ \\
Discharged alive & No & Yes & Yes & \\
SVC size, cm, mean (SD) & & & & \\
$\quad$ Length & 52 & 59 & 52 & $64(13)$ \\
$\quad$ Diameter/lateral & 14 & 14 & 13 & $15(3)$ \\
$\quad$ Area & 224 & 196 & 221 & $189(72)$ \\
Thrombotic risk (previous DVT, malignancy, etc) & No & Yes & No & $2(5 \%)$ \\
BSA, m ${ }^{2}$, mean (SD) & 2.14 & 2.05 & 1.66 & $2.09(0.26)$ \\
RVAD peak flow, L, mean (SD) & 3.9 & 6 & 4.2 & $4.5(0.8)$ \\
RVAD peak rate, RPM, mean & 3900 & 5200 & 4500 & 4644 \\
\hline
\end{tabular}

$S V C$, Superior vena cava; $R V A D$, right ventricular assist device; $S D$, standard deviation; $D V T$, deep vein thrombosis; $B S A$, body surface area; $R P M$, rate per minute.

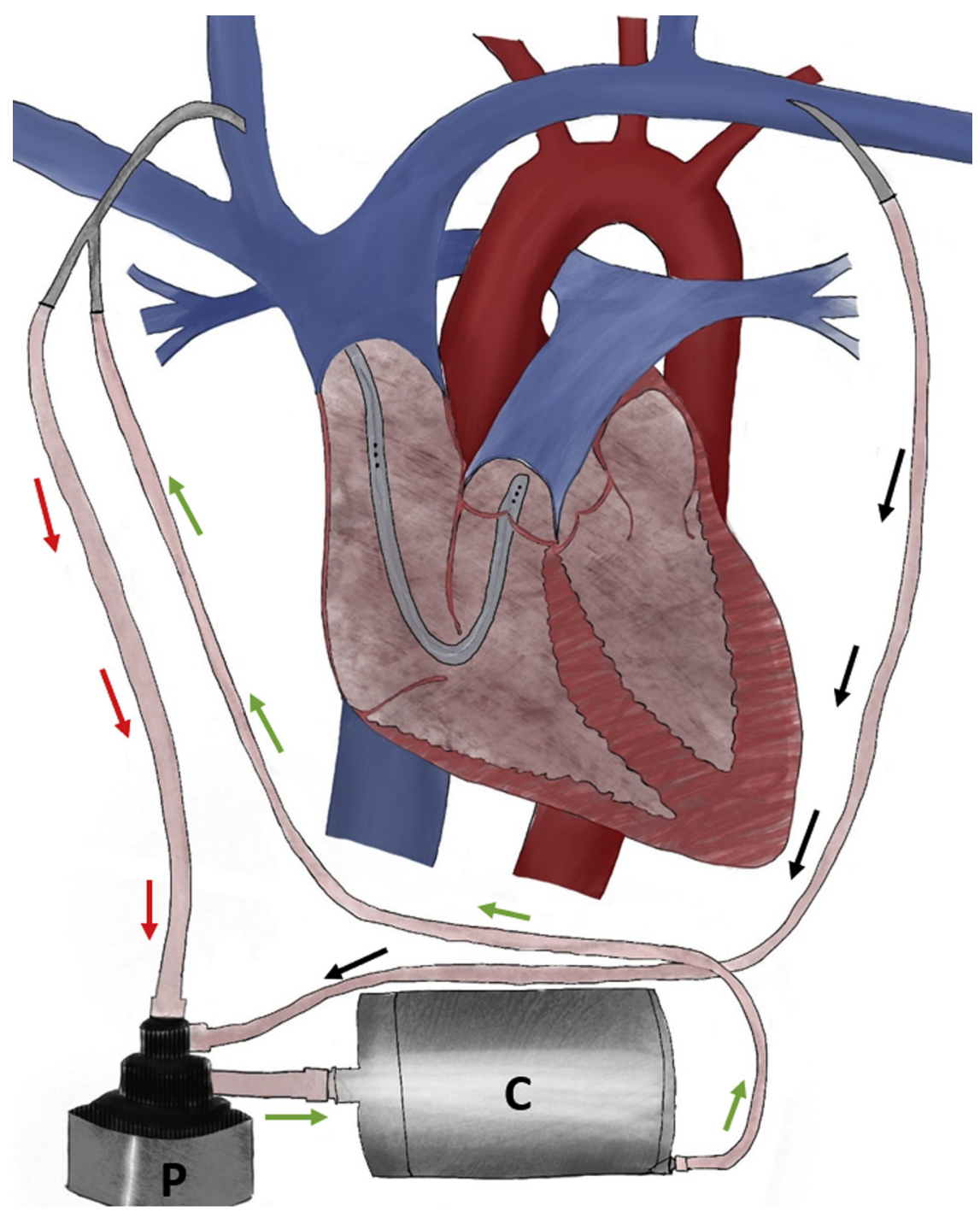

FIGURE 1. ProtekDuo dual-lumen percutaneous right ventricular assist device in the right internal jugular vein. Additional cannula (black arrow) inserted in the left subclavian vein to reduce venous congestion from superior vena cava syndrome. Outflow cannula (red arrow) is connected to an external pump $(P)$ that feeds into external circuit $(C)$ to circulate venous blood through inflow cannula (green arrow). 




VIDEO 1. Traditionally, right ventricular (RV) failure has presented a significant problem in our cardiac surgical patients, particularly those who have undergone orthotopic heart transplant, post-LVAD, or after complex cardiac procedures. RV failure is a challenging clinical diagnosis and equally challenging to treat. Medical management of RV failure is very expensive and does not reverse RV failure in the way that right ventricular assist devices (RVADs) can. Surgical RVADs, however, increases morbidity as weaning sometimes requires that the median sternotomy remains open; in addition, patients are often connected to ventilator and are not able to ambulate. Hence, percutaneous RVADs via right internal jugular vein such as the ProtekDuo has been widely used at our institution due to decreased mortality and improved weaning outcomes in various clinical scenarios. Percutaneous RV support also allows for upright positioning and patients can ambulate. The only complication concerning this approach is that, in potentially smaller patients or those with a smaller-sized central vein, we have seen obstruction leading to venous congestion of the face, neck, and upper extremities consistent with SVC syndrome. Fortunately, we have found that reducing venous congestion via drainage from the left subclavian (or IJ) is sufficient to resolve SVC syndrome and allow patients to remain on support as they recover from RV failure. Video available at: https://www.jtcvs.org/article/S2666-2507 (20)30694-5/fulltext.

opted to proceed with comfort care and withdrawal of support. She soon died on biventricular support.

\section{Patient 2}

A 40-year-old male patient with (non)ischemic cardiomyopathy status post durable LVAD developed acute RV and respiratory failure 1 day after orthotopic heart transplant, indicating a need for RV support with a 31-Fr DLC. Following this, he developed distension of neck veins and facial edema concerning for SVC syndrome. His medical history was positive for a thrombus in the RIJ 5 years previously that resolved after a course of oral anticoagulation. Postoperative computed tomography scan did not visualize an SVC or internal jugular thrombus, and he was diagnosed with SVC syndrome based on the clinical findings and absence of anatomical obstruction on imaging. Venous congestion was reduced using the technique described previously at the patient's bedside and led to quick improvement in symptoms. He was weaned from RVAD support after 14 days and later discharged after an uncomplicated remaining hospital course. The patient continues to have optimal cardiovascular function nearly 2 years after his heart transplantation.

\section{DISCUSSION}

In this case series, we found that SVC syndrome is a serious, previously undescribed complication of percutaneous RVAD via RIJ for patients with refractory RV failure (Video 1). Although symptoms, which include cyanosis, edema, and neurologic sequelae, are a result of venous occlusion, data show that only a subset of patients with obstruction of the SVC develop the syndrome. ${ }^{5}$

We believe that anatomic variability in SVC length as well as cannula size choice are likely underlying contributors to the development of iatrogenic SVC syndrome. While a 31-Fr cannula allows for more adequate flows, maximum RV recovery, and longer duration of support compared with 29-Fr cannulas, we believe that this size cannula led to venous congestion in our smaller patients and/or those with comparatively shorter vessel length (patient 1 ) or deep vein thrombosis risk (patient 2). Other metrics such as diameter (anteroposterior and lateral) and area did not demonstrate a correlation between size and SVC syndrome secondary to obstruction.

Fortunately, we developed an easy technique (Figure 1) to drain venous blood back into the circuit that rapidly resolves symptoms and permits patients to remain on RVAD support long enough for RV recovery. Although SVC syndrome is a potentially life-threatening condition, early recognition of clinical symptoms and imaging is necessary to prevent progression. Preoperative vessel measurements may be warranted before undergoing placement with a 31-Fr RVAD cannula to prevent SVC syndrome in some patients.

Consent was obtained from patients in this study.

Special thanks to Nathan J. Smith, MD, Zachary R. Laste, MD, Michael T. Cain, MD, Emily Neumann, and Courtney Goulet for contributions to this report.

\section{References}

1. Badu B, Cain MT, Durham LA III, Joyce LD, Sundararajan S, Gaglianello N, et al A dual-lumen percutaneous cannula for managing refractory right ventricular failure. ASAIO J. 2020;66:915-21.

2. Nicolais CD, Suryapalam M, O'Murchu B, Bashir R, O'Neill BP, Alvarez R, et al. Use of Protek Duo Tandem Heart for percutaneous right ventricular support in various clinical settings: a case series. J Am Coll Cardiol. 2018;71(11_suppl): A1314.

3. Ravichandran AK, Baran DA, Stelling K, Cowger JA, Salerno CT. Outcomes with the Tandem Protek Duo dual-lumen percutaneous right ventricular assist Device. ASAIO J. 2018;64:570-2.

4. Coromilas EJ, Takeda K, Ando M, Cevasco M, Green P, Karmpaliotis D, et al. Comparison of percutaneous and surgical right ventricular assist device support after durable left ventricular assist device insertion. J Card Fail. 2019;25:105-13.

5. Tzifa A, Marshall AC, McElhinney DB, Lock JE, Geggel RL. Endovascular treatment for superior vena cava occlusion or obstruction in a pediatric and young adult population: a 22-year experience. J Am Coll Cardiol. 2007;49: 1003-9. 